\title{
Clinical outcomes of the simultaneous bilateral percutaneous nephrolithotomy (PCNL) in patients with kidney stones: A prospective cohort study
}

\author{
Mohammad Reza Darabi ${ }^{1}$, Salman Soltani ${ }^{2}$, Alireza Akhavan Rezayat ${ }^{2}$, Masoud Yousefi ${ }^{3}$, Mehdi Kashefi ${ }^{3}$, \\ Mahmoud Tavakkoli ${ }^{2}$, Shabnam Mohammadi ${ }^{4}$
}

${ }^{1}$ M.D., Professor of Urology, Department of Urology, Faculty of Medicine, Mashhad University of Medical Sciences, Mashhad, Iran

${ }^{2}$ M.D., Assistant Professor of Urology, Department of Urology, Faculty of Medicine, Mashhad University of Medical Sciences, Mashhad, Iran

${ }^{3}$ M.D., Resident of Urology, Department of Urology, Faculty of Medicine, Mashhad University of Medical Sciences, Mashhad, Iran

${ }^{4}$ Ph.D., Assistant Professor of Anatomy, Department of Basic Sciences, Faculty of Medicine, Gonabad University of Medical Sciences, Gonabad, Iran

Type of article: Original

\begin{abstract}
Background: Urinary tract stones is one of the most frequent medical emergencies which leads to lifethreatening complications, namely obstructive uropathy as well as renal failure in some situations. Previously, bilateral stones were treated with either open surgery or percutaneous nephrolithotomy (PCNL). However, these treatment options were associated with lengthy operation time, need for more anesthesia, further bleeding, and long hospitalization. Therefore, much effort has been made to treat both sides simultaneously.

Objective: The aim of this study was an attempt to prospectively review cases undergoing simultaneous bilateral PCNL in terms of the therapy outcomes, complications, and consequences.

Methods: In this prospective cohort study, 39 adult patients with bilateral renal stones were randomly recruited at Imam Reza hospital in Mashhad, Iran between January 2016 and January 2017. Adult patients with bilateral renal stones were included in this study. Exclusion criteria were as follows: Patients with severe heart or lung disease, patients with coagulation disorders, pregnant women, and cases with any contraindications for general anesthesia. After insertion of bilateral ureteral catheters, all patients underwent simultaneous bilateral PCNL in prone position. Transureteral lithotripsy was performed for patients with ureteral stones. The surgery was initially carried out on the symptomatic side and then iterated on the remaining kidney. Major complications including bleeding, fever, pain, urine leakage, and residual stones were recorded. SPSS software was used for data analysis. Data were expressed as percentage and mean \pm SD. P value less than 0.05 was considered significant.

Results: A total of 39 patients (27 males with mean age of 37.6 years and 12 females with mean age of 45.7 years) were studied. As many as $15(38 \%)$ patients received a unilateral nephrostomy. Three underwent totally tubeless surgery. Bleeding (41.0\%) was the most common complication, followed by residual stones $(20.5 \%)$ and fever $(20.5 \%)$, urine leakage $(15.3 \%)$, pain $(12.8 \%)$, blood transfusion $(2.5 \%)$ and colon perforation $(2.5 \%)$.

Conclusions: It was concluded that simultaneous bilateral PCNL was not associated with higher morbidity than the unilateral method.

Keywords: Bilateral calculi, Complications, Percutaneous nephrolithotomy, PCNL, Renal stones
\end{abstract}

\section{Corresponding author:}

Assistant Professor Dr. Salman Soltani, Department of Urology, Faculty of Medicine, Mashhad University of Medical Sciences, Mashhad, Iran. Tel: +98.5138022553, Fax:+98. 5138022553, Email: SoltaniS@mums.ac.ir Received: May 04, 2017, Accepted: December 23, 2017, Published: February 2018

iThenticate screening: October 07, 2017, English editing: January 06, 2018, Quality control: February 10, 2018

This article has been reviewed / commented by three experts

(C) 2018 The Authors. This is an open access article under the terms of the Creative Commons Attribution-NonCommercialNoDerivs License, which permits use and distribution in any medium, provided the original work is properly cited, the use is non-commercial and no modifications or adaptations are made. 


\section{Introduction}

Urinary tract stones (UTS) is well-known as the most common urological condition (1-3). Given the high incidence of the disease (about $12 \%$ in men and $7 \%$ in women), its treatment has drawn much attention across the world (1-3). UTSs can be either unilateral or bilateral. Of note, bilateral renal stones which has risk factors such as obstructions in urinary tract systems and metabolic disorders are associated with life-threatening complications such as obstructive uropathy and renal failure, hence, peculiar consideration of any immediate intervention is necessary (1, 4). Over the past years, the symptomatic side, or the side with larger stone burden did initially undergo the treatment of choice- open surgery or percutaneous nephrolithotomy (PCNL). Recently, the simultaneous bilateral PCNL is taken into account in an attempt to overcome the consequences of that condition, namely the lengthy operation time, more need of anesthesia, high risks of further bleeding, and long hospitalization (5-7). Considering a sharp drop in patient cost, shorter length of hospital stay, less need for sedative agents and blood transfusion, rapid return to normal life functioning and favor for the management of renal stones with large size, PCNL seems be safe, with fewer complications and less general anesthesia as compared to open surgery (8-10). Stone disease prevalence rate in Iran, a country of great variation in geography and climate, is $5.7 \%$, and its incidence is $145 / 100,000$ (11). In a study that was conducted in Taiwan, safety and efficacy of PCNL were investigated in patients with a mean age of 54.4 years. Stone free rate was $78.3 \%$. Average length of hospitalization was 3.6 days and the need for blood transfusion was $2.4 \%$. Fever in 104 patients and urosepsis in 13 patients were found. Congestive heart failure was observed in 1 patient and pneumomediastinum in 1 patient. In addition, pneumothorax in 2 patients, hydrothorax in 5 patients, hemothorax in 2 patients and pulmonary edema in 1 patient were reported (12). In another study, 10.9\% of patients required a blood transfusion after PCNL. Postoperative fever was observed in $2.8 \%$ of cases (13). Efficacy and safety of simultaneous and stage PCNL as two methods of PCNL were investigated in other research. The results showed that $94.1 \%$ stone free rate was in the staged group and $92.3 \%$ in the simultaneous group. No severe complication was observed in all patients. Length of hospitalization and cost of treatment was more in the staged group than the simultaneous group (14). In a systematic review, outcome and efficacy of simultaneous bilateral percutaneous nephrolithotomy were investigated. Mean stone free rate was $92.4 \%$. The mean operation time was 2 hours and 51 minutes. The mean hospitalization was 3.9 days. Average of complications was $23.4 \%$ and the most common complication was fever (15). The aim of this study was to determine the outcomes and complications of those cases undergoing simultaneous bilateral PCNL.

\section{Material and Methods}

In this prospective cohort study, 39 patients with bilateral renal stones were recruited at Imam Reza hospital in Mashhad, Iran between January 2016 and January 2017, and underwent simultaneous bilateral PCNL. Adult patients with bilateral renal stones were included in this study. Exclusion criteria were as follows: patients with severe heart or lung disease, patients with coagulation disorders, pregnant women, and cases with any contraindications for general anesthesia. Of 41 patients with bilateral stones, two were excluded due to inaccessibility of the renal stones. Single stones in both kidneys were found in 32 patients. Seven patients showed incomplete staghorn stone in one kidney while two developed this type of stone bilaterally. Cases of urinary tract infection (UTI) were treated 48 hours before surgery based on the results of U/C and U/A. A $1 \mathrm{~g}$ cefazolin plus $80 \mathrm{mg}$ gentamicin for patients with no UTI. Under the induction of general anesthesia, two 5-Fr ureteral catheters with different colors were inserted at the start of the procedure after cystoscopy. In the presence of ureteral stones, TUL was initially carried out and subsequently, ureteral catheters were placed. Thereafter, all patients underwent simultaneous bilateral PCNL in prone position. The PCNL was first performed on the symptomatic side and on the other side subsequently. Tract was dilated to a diameter of 28- or 30- French Amplatz sheath under fluoroscopic control. When the PCNL was completed on one side, the same procedure was iterated on the remaining kidney. At the completion of the surgery, fluoroscopy was performed to ensure thorough removal of the stone fragments. Bilateral nephrostomy drainage was reserved for 21 patients. However, the urinary catheters were clamped for 4-6 hours and then removed upon the stage of transfer to the division. Demographic data, complications, success rate, undesirable effects, and possibility of open surgery were explored. Those with a prior renal stone surgery or pyeloplasty showed difficult percutaneous access in the routine dorsal approach to the stone, owing to scar and fibrous tissues. Thus, difficulty in tract dilatation was observed in these patients. Throughout PCNL, small stones were removed with forceps. This study was approved by the ethic committee of Mashhad University of Medical Sciences. SPSS software was used for data analysis. Data was expressed as percent and mean $\pm \mathrm{SD}$. P value less than 0.05 was considered significant. 


\section{Results \\ 3.1. General findings}

A total of 39 eligible patients was identified, with $27(69 \%)$ males and $12(31 \%)$ females. Age of patients was $41.07 \pm 19$ years. Mean age in male patients was 37.6 years while the mean age in females was 45.3 years. On placing of a nephroscope, a pneumatic lithotripsy was used to break the stones in all patients. Three cases of obesity were observed $(>100 \mathrm{~kg})$. All steps of PCNL were performed under fluoroscopic guidance which was continued postoperatively. Patients were transferred to the division of urology and underwent a broad-spectrum antibiotic treatment (a combination of $1 \mathrm{gr}$ cefazolin and $80 \mathrm{mg}$ gentamicin every 8 hours) except for those with elevated serum levels of $\mathrm{Cr}$. This antibiotic regimen was maintained following discharge for one month. As many as 19 cases had prior history of unilateral renal stone surgery, while three had previous stone removal on both sides. Moreover, there was only one patient who underwent unilateral pyeloplasty before PCNL. Sixteen patients had been treated with one or two extracorporeal shock wave lithotripsy (ESWL) sessions for the treatment of bilateral stones that failed to thoroughly remove the stones. Three patients with bilateral kidney stones had concurrent ureteral stones, thus, transureteral lithotripsy (TUL) was also conducted in such cases. Patients were matched in terms of age, sex, history of surgery, and drug use, which may have a confounding state. All patients were evaluated with laboratory tests including serum creatinine (Cr), blood urea nitrogen (BUN), fasting blood sugar (FBS), uric acid, potassium $(\mathrm{K})$, sodium $(\mathrm{Na})$, calcium $(\mathrm{Ca})$ with a complete blood count $(\mathrm{CBC})$ before the surgery. The levels of $\mathrm{Cr}$, uric acid, $\mathrm{Ca}$ were also tested in the 24-hour urine sample. Metabolic causes had not been reported in any patient. Urinalysis (U/A) and urine culture (U/C) was carried out in all subjects. Ultrasound examination as well as CT scan were performed in eight patients before referral to our center. In cases of normal levels of $\mathrm{Cr}$, an intravenous pyelogram (IVP) was used to evaluate renal anatomy and identify kidney stones. Two patients were examined by employing spiral CT scanning because of increased Cr levels between 2-2.5 mg/dl. In 15 patients, a nephrostomy catheter was inserted only on one side which was at risk of renal rupture or residual stones. Accordingly, a tubeless approach was adopted on the other side in these subjected. Of 39 patients, there were three cases of totally tubeless PCNLs who had no significant load of residual stone under nephroscopy. Following the PCNL surgery, patients were transferred to the recovery room, and ultrasound examination of kidney, ureter and bladder (KUB) was performed for all of them the following day. Stone particles were reported in eight subjects, half of whom with no clinical value. The other half underwent another ESWL. Those patients whose preoperative $\mathrm{Cr}$ levels were high, displayed a decrease on the first week after the surgery and a normal level during follow-up thereafter. Three patients reached the normal levels of $\mathrm{Cr}$ one month following the PCNL despite an initial increase in its serum levels on the first week. Stone free rates (SFR) was $79.56 \%$. Mean operative time was $50 \mathrm{~min}$ (45-90 min).

\subsection{Complications}

Bleeding $(41.0 \%)$ was the most common complication of simultaneous bilateral percutaneous nephrolithotomy, followed by residual stone $(20.5 \%)$ and fever $(20.5 \%)$, as shown in Table 1 . Seventeen patients required another PCNL one month after the bilateral PCNL. In patients presenting bleeding, they were advised rest and fluid administration. Blood transfusion was prescribed in one subject and then completed with rest, painkillers and additional protective and supportive actions. We encountered six cases of urine leakage around the nephrostomy tube, which was improved by applying conservative treatment. Two of whom, for instance, received the insertion of a double-J catheter. One week after the PCNL, five patients developed pain as well as abdominal sensitivity that underwent renal ultrasonography. The results presented a mere urinoma, which was recovered with medical treatment besides sonographic control. Colon perforation occurred in one case on the right side. Following the removal of the nephrostomy tube, fecal egression was noticed around the nephrostomy area. Therefore, a double-J catheter was used and drainage catheters remained in fistula. The patient was ordered to be NPO for 5 consecutive days along with broad-spectrum antibiotics and surgery consultation. When the intestinal fistula closed, the patients were later discharged in good condition. One month later, a double-J catheter was removed. Cases of significant residual stones were treated with ESWL one month after the PCNL. Three days following hospital discharge, two presented with colic pains caused by residual stones that had entered the ureter. In these cases, they underwent TUL as well as double-J catheter placement. Regular diet was commenced for all patients after the PCNL. On day 2 following the surgery, they were discharged while their nephrostomy catheter was brought out. Similarly, the ureteral catheters were removed on day 3. Patients undergoing bilateral tubeless PCNL were discharged following the day of surgery. On the next day, their ureteral catheters were removed. It has been found that the mean duration of hospitalization of patients undergoing bilateral PCNL with nephrostomy tube placement was two days, which reduces to one day in tubeless cases. 
Table 1. Frequency of complications of simultaneous bilateral percutaneous nephrolithotomy

\begin{tabular}{|l|l|}
\hline Complications & Patients $(\mathrm{n}=39), \mathrm{n}(\%)$ \\
\hline Fever & $8(20.5)$ \\
\hline Urosepsis & - \\
\hline Stricture & - \\
\hline Pain & $5(12.8)$ \\
\hline Urine leakage & $6(15.3)$ \\
\hline Residual stone & $8(20.5)$ \\
\hline Bleeding & $16(41.0)$ \\
\hline Blood transfusion & $1(2.5)$ \\
\hline Colon perforation & $1(2.5)$ \\
\hline Drop hemoglobin & - \\
\hline Hematuria & - \\
\hline Cardiovascular complications & - \\
\hline Pulmonary complications & - \\
\hline
\end{tabular}

\section{Discussion}

Our findings indicated that $38 \%$ of patients received a unilateral nephrostomy. Three underwent totally tubeless surgery. Residual stones were not found in 31 cases. Half of residual stones lacked clinical value and four patients were treated with an additional extracorporeal shock wave lithotripsy session. Urine leakage occurred in the nephrostomy area in 6 patients. One patient suffered from colon perforation on the right side. Blood transfusion was required in one case. Three patients had slightly elevated levels of $\mathrm{Cr}$ after surgery, which was followed by a normal level. This was reported by previous studies, as well. For example, in a study from Pakistan by Shaikh, one out of 10 patients showed an increase in serum $\mathrm{Cr}$ levels that returned to normal at follow-up $(16,17)$. Moreover, this review identified 8 cases with residual stones, of which only four were of clinical value and considered the need for another ESWL; to put it differently, the stone-free rate was approximately $79.56 \%$. Several studies revealed different percentages in this regard $(12-15,20)$. In this study, only one was found with colon perforation and fistula that resolved with the insertion of a urethral catheter and conservative treatment. Similarly, Multescu et al. observed a case of fistula in 6 patients who could be managed by means of conservative treatment (16). The incidence of nonsignificant bleeding was $41 \%$ in this study. One patient $(2.5 \%)$ required blood transfusions, which was consistent with the findings of Dushinsky et al. (3\%) yet a far cry from the findings of Silverstein et al. $(38.6 \%)(18,19)$. In a study that was conducted in Taiwan, safety and efficacy of PCNL were investigated in patients with mean age of 54.4 years. Stone free rate was $78.3 \%$. Average length of hospitalization was 3.6 days and the need for blood transfusion was $2.4 \%$. Fever in 104 patients and urosepsis in 13 patients were found. Congestive heart failure was observed in 1 patient and pneumomediastinum in 1 patient. In addition, pneumothorax in 2 patients, hydrothorax in 5 patients, hemothorax in 2 patients and pulmonary edema was reported in 1 patient (12). In our study, urosepsis, cardiovascular and pulmonary complications were not observed. Stone free rate was $79.56 \%$ and the need for blood transfusion was $2.56 \%$. Fever was observed in 8 patients. In another study in China, $10.9 \%$ of patients required blood transfusion after PCNL. Postoperative fever was observed in $2.8 \%$ of cases (13). Our findings showed need for blood transfusion in $2.56 \%$ and postoperative fever in $20.51 \%$. In another study in China, efficacy and safety of simultaneous and stage PCNL as two methods of PCNL were compared in 102 patients. The results showed that a 94.1\% stone free rate was in the staged group and $92.3 \%$ in the simultaneous group. No severe complication was observed in all patients. Length of hospitalization and cost of treatment was more in the staged group than in the simultaneous group (14). In a study in India, tubeless simultaneous bilateral percutaneous nephrolithotomy was performed in 85 patients with an average age of 45.7 years. Average size of stones was $299 \mathrm{~mm} 2$. The success rate was $95.2 \%$ and mean drop hemoglobin was $1.11 \mathrm{gm}$ per patient and $10.5 \%$ required blood transfusion. The mean hospitalization was 69.6 hours. Complications included urosepsis, kidney damage that lead to dialysis, pneumonia and hydrothorax (20). In our cases, the reduction of hemoglobin $1.41 \mathrm{~g} / \mathrm{l}$ was observed. Need for blood transfusion was $2.56 \%$. Pulmonary complications, kidney damage and urosepsis were not observed and the hospitalization was lower than this study. In a systematic review, outcome and efficacy of simultaneous bilateral percutaneous nephrolithotomy were investigated. Mean stone free rate was $92.4 \%$. The mean operation time was 2 hours and 51 minutes. The mean hospitalization was 3.9 days. Average complications percentage was $23.4 \%$ and the most common complication was fever (15). In our study, hospitalization and mean operative time was lower than in this research. Moreover, the most common complication was low bleeding. However, these studies included a limited 
number of patients and may require longer follow-up periods to better determine the strengths or limitations of each procedure.

\section{Conclusions}

Simultaneous bilateral PCNL may not be associated with increased morbidity than the unilateral method. Patients with bilateral stones who cannot undergo the unilateral or bilateral ESWL, or the ESWL failed to be successful, are advised to consider simultaneous bilateral PCNL. However, they should be fully informed of the procedure and any possible complications. In this study, we came to the conclusion that the simultaneous bilateral PCNL did not show inconsistent outcomes after careful monitoring of the symptoms, complications, and postoperative results. To sum up, patients afflicted with bilateral stones should be treated with other minimally invasive procedures; as decisive treatment, the simultaneous bilateral PCNL is recommended as showing the same risk of complication as the unilateral PCNL.

\section{Acknowledgments:}

The authors thank staff members of the Urology Department of the Medical School at the University of Mashhad, Iran.

\section{Conflict of Interest:}

There is no conflict of interest to be declared.

Authors' contributions:

All authors contributed to this project and article equally. All authors read and approved the final manuscript.

\section{References:}

1) Leslie SW, Bhimji SS. Calculi, Renal. StatPearls. Treasure Island FL: StatPearls Publishing LLC. 2017. PMID: 28723043.

2) Raheem OA, Khandwala YS, Sur RL, Ghani KR, Denstedt JD. Burden of Urolithiasis: Trends in Prevalence, Treatments, and Costs. Eur Urol Focus. 2017; 3(1): 18-26. doi: 10.1016/j.euf.2017.04.001. PMID: 28720363.

3) Arrabal-Martín M, Cano-García MC, Arrabal-Polo MÁ, Dominguez-Amillo A, Canales-Casco N, de la Torre-Trillo J, et al. Etiopathogenic factors of the different types of urinary litiasis. Archivos espanoles de urologia. 2017; 70(1): 40-50. PMID: 28221141.

4) Stoller ML, Bolton DM. Urinary stone disease. IN: Emil A Tanagho, Jack W McAninch, Smiths General Urology. 15th editin. McGraw-Hill. Middle East edition, Lebanon. 2000; 291-320.

5) Lingeman JE, Lifshitz DA, Evan AP. Surgical management of urinary lithiasis. Campblle Urology. 8th ed. Philadelphia-Pennsylvania: Saunders, 2002; 4: 3362-96.

6) Al-Kohlany KM, Shokeir AA, Mosbah A, Mohsen T, Shoma AM, Eraky I, et al. Treatment of complete staghorn stones: a prospective randomized comparison of open surgery versus percutaneous nephrolithotomy. J Urol. 2005; 173(2): 469-73. doi: 10.1097/01.ju.0000150519.49495.88. PMID: 15643212.

7) Holman E, Khan AM, Pasztor I, Tóth C. Simultaneous bilateral compared with unilateral percutaneous nephrolithotomy. BJU Int. 2002; 89: 334-8. doi: 10.1046/j.1464-4096.2001.01521.x. PMID: 11872019.

8) Wang CJ, Chang $\mathrm{CH}$, Huang SW. Simultaneous bilateral tubeless percutaneous nephrolithotomy of staghorn stones: a prospective randomized controlled study. Urol Res. 2011; 39(4): 289-94. doi: 10.1007/s00240-010-0342-x. PMID: 21161650.

9) Preminger GM, Assimos DG, Lingeman JE, Nakada SY, Pearle MS, Wolf JS Jr. Chapter 1: AUA guideline on management of staghorn calculi: diagnosis and treatment recommendations. J Urol. 2005; 173(6): 1991 2000. doi: 10.1097/01.ju.0000161171.67806.2a. PMID: 15879803.

10) Ganpule AP, Vijayakumar M, Malpani A, Desai MR. Percutaneous nephrolithotomy (PCNL) a critical review. Int J Surg. 2016; 36: 660-4. doi: 10.1016/j.ijsu.2016.11.028. PMID: 27856356.

11) Pourmand G, Pourmand B. Epidemiology of Stone Disease in Iran. In Urolithiasis. Springer London. 2012: 85-7. doi: 10.1007/978-1-4471-4387-1_11.

12) Lai WH, Jou YC, Cheng MC, Shen $\overline{C H}$, Lin CT, Chen PC, et al. Tubeless percutaneous nephrolithotomy: Experience of 1000 cases at a single institute. Urological Science. 2017; 28(1): 23-6. doi: 10.1016/j.urols.2016.04.004. 
13) Tefekli A, Karadag MA, Tepeler K, Sari E, Berberoglu Y, Baykal M, et al. Classification of percutaneous nephrolithotomy complications using the modified clavien grading system: looking for a standard. European urology. 2008; 53(1): 184-90. doi:10.1016/j.eururo.2007.06.049. PMID: 17651892.

14) Shen PF, Liu N, Wei WR, Xu P, Li S, Luo YH, et al. Simultaneous ureteroscopic lithotripsy and contralateral percutaneous nephrolithotomy for ureteral calculi combined with renal staghorn calculi. International Journal of Urology. 2015; 22(10): 943-8. doi: 10.1111/iju.12862. PMID: 26149937.

15) Jones P, Dhliwayo B, Rai BP, Mokete M, Amitharaj R, Aboumarzouk OM, et al. Safety, Feasibility, and Efficacy of Bilateral Synchronous Percutaneous Nephrolithotomy for Bilateral Stone Disease: Evidence from a Systematic Review. Journal of Endourology. 2017; 31(4): 334-40. doi: 10.1089/end.2016.0851. PMID: 28326800.

16) Shaikh AH, Khalid SE, Nabi N. Safety and efficacy of tubeless percutaneous nephrolithotomy. J Pak Med Assoc. 2007; 57(12): 584-6. PMID: 18173039.

17) Proietti S, Sortino G, Giannantoni A, Sofer M, Peschechera R, Luciani LG, et al. Single-session supine bilateral percutaneous nephrolithotomy. Urology. 2015; 85(2): 304-10. doi: 10.1016/j.urology.2014.10.036. PMID: 25623671.

18) Dushinski JW, Lingeman JE. Simultaneous bilateral percutaneous nephrolithotomy. The Journal of urology. 1997; 158(6): 2065-8. PMID: 9366313.

19) Silverstein AD, Terranova SA, Auge BK, Weizer AZ, Delvecchio FC, Pietrow PK, et al. Bilateral renal calculi: assessment of staged v synchronous percutaneous nephrolithotomy. Journal of Endourology. 2004; 18(2): 145-51. doi: 10.1089/089277904322959770. PMID: 15072621.

20) Pillai S, Mishra D, Sharma P, Venkatesh G, Chawla A, Hegde P, et al. Tubeless simultaneous bilateral percutaneous nephrolithotomy: Safety, feasibility and efficacy in an Indian setting. International Journal of Urology. 2014; 21(5): 497-502. doi: 10.1111/iju.12352. PMID: 24286445. 